\title{
LIE SYMMETRIES OF DIFFERENTIAL EQUATIONS BY COMPUTER ALGEBRA
}

\author{
Mehmet Can \\ Istanbul Technical University Mathematics Department \\ Maslak 80626 Istanbul TURKIYE
}

\begin{abstract}
In this paper we restrict ourselves to Lie point symmetries an applications to the fourth order generalized Burgers equation GBE4. Using computer programs under the computer algebra package MATHEMATICA we find a three dimensional solvable Lie algebra of point symmetries of the GBE4 equation. The similarity reductions due to these symmetries have also been obtained.
\end{abstract}

\section{Introduction}

The idea of applying Lie group theory to solve differential equations is as old as the Lie group theory itself [1]. An extensive literature exist on this subject but untill quite recently, group theory has in this respect been unused. The reason may be several misconceptions in the minds of potential users of group theory, such as

i) Is is as difficult to find symmetry group of an equation as to solve it,

ii) Group theory only provides randomly occuring particular solutions,

iii) Group theory is only useful for linear equations [2].

The symmetry group of a system of differential equations is, roughly speaking, a group of transformations of independent and dependent variables leaving the set of all solutions invariant. Once the symmetry group of a system of equations is known, it can be used to generate new solutions from the old ones, often interesting ones from trivial ones. It can be used to classify solutions into conjugacy classes and to classify and simplify differential equations. An importent application is the symmetry reduction of an ordinary differential equation (ODE) to a lower order one, the reduction of a partial differential equation (PDE) to one with fewer independent variables.

\section{Classical Lie Symmetries of Differential Equations}

Now we introduce the classical Lie symmetries or Lie point symmetries of the partial dif-ferential equations which can be obtained through the Lie group method of infinitesimal transformations, originally developed by Sophus Lie [1]. Thoug the method is entirely algorithmic, it often involves a large amount of tedious algebra and auxiliary calculations which are virtually unmanagable manually.

During the last two decades a change has occured in applied mathematics that is even more severe than the introduction of computers for performing numerical calculations about forty years ago. It means that large computers have rendered it feasible to perform analytical calculations automatically as well, although the idea of mechanizing analytical calculations is already more than 100 years old. 
The most important general purpose computer algebra systems available today are . Macsyma by Mathlab Group at MIT, Reduce by A. C. Hearn at the Rand Corparation, Maple by B. Char at Waterloo, mu-Math by D. R. Stoutmeyer of Software House in Honolulu, SMP (Mathematica) by S. Wolfram and Scratchpad II by R. D. Jenks and D. Yun at IBM.

Typically a computer algebra system provides modules for performing basic operations like simplification, differentiation, integration, factorization, etc. These algorithms are the building blocks for any other packages which may be developed by the user for special applications.

The availability of these computer algebra systems has a particular strong influence on those areas of applied mathematics where large analytical manipulations are necessary for obtaining a certain result. Applying a computer algebra system means to become accustomed to a completely new working style for the symmetry analysis of differential equations.

Althoug the concept of symmetry of a differential equation was introduced by Sophus Lie at the end of the last century while he was searching a general theory of solving differential equations, it did not receive the proper attention for a long time. The reason is quickly recognized if we try to apply it to specific problems. To find the symmetry group of a differential equation almost always requires tremendeus algebraic calculations. Especially in partial differential equations, they often assume such proportions that they can not be performed in the conventional way.

The largest number of algebraic calculations is required for solving the so called determining system of linear partial differential equations which may have a simple structure; however as it can be observed in the example of GBE4, it may comprize several dozens or even hundreds of equations. The solution algorithm for these determining system, is the heart of the symmetry packages. In addition to providing the symmetry generators of the full symmetry group, its structure is also determined automatically and communicated to the user in terms of its commutator table.

In order to facilitate the determination of the classical Lie symmetries, we use the package Lie[ ] of G. Baumann [3] under the computer algebra software MATHEMATICA and find a three dimensional solvable Lie algebra of infinitesimal transformations. When we used SPDE package in the computer algebra software REDUCE [4] for the same PDE, we have got a different result. The result given by this program was wrong. There was a misplaced sign. We also tried the SYMMAN package of Vorob'ev [5] under the computer algebra packege MATHEMATICA again, but it gave only two of the three symmetries.

\subsection{Invariance of a $\mathrm{PDE}$}

In this subsection we apply infinitesimal transformations to the construction of solutions of partial differential equations. We will show that infinitesimal criterion for invariance of partial differential equations leads directly to an algorithm to determine infinitesimal generators $\mathrm{X}$ admitted by given partial differential equations. Invariant surfaces of the corresponding Lie group of point transformations lead to invariant solutions. These solutions are obtained by solving partial differential equations with fewer independent variables than the given PDE's. 
We represent a $\mathrm{k}$ th order partial differential equation by

$$
F\left(x, u, u_{1}, u_{2}, \cdots, u_{k}\right)=0,
$$

where $x=\left(x_{1}, x_{2}, \ldots, x_{n}\right)$ denotes $n$ independent variables, $u$ denotes the coordinate corresponding to the dependent variable, and $u_{j}$ denotes the set of coordinates corresponding to all $j$ th order partial derivates of $u$ with respect to $x$; the coordinate $u$; corresponding to $\partial^{j} u /\left(\partial x_{i_{1}} \partial x_{i_{2}} \ldots \partial x_{i_{j}}\right)$ is denoted by $u_{i_{i_{2}-1},}, i_{j}=1,2, \ldots, n$ for $j=1,2, \ldots, k$. In terms of the coordinates $x, u, u_{1}, \ldots, u_{k}$, equation (1) becomes and algebraic equation which defines a hypersurface in $\left(x, u, u_{1}, \ldots, u_{k}\right)$-space.

We asume that the partial differential equation (1) can be written in solved form in terms of some $l$ th order partial derivative of $u$ :

$$
F\left(x, u_{1} u_{1}, u_{2}, \cdots, u_{k}\right)=u_{i_{1}, i_{2}}, \ldots, i_{i}-f\left(x, u_{1} u_{1}, u_{2}, \cdots, u_{k}\right)=0,
$$

where $f\left(x, u_{1}, u_{2}, \cdots, u_{k}\right)$ does not depend on $\quad u_{i_{1}, i_{2}, \ldots, i_{l}}$.

Now we are going to give a criterion for the invariance of a PDE [6].

Theorem. Let

$$
X=\xi_{i}(x, u) \frac{\partial}{\partial x_{i}}+\eta(x, u) \frac{\partial}{\partial u}
$$

be the infinitesimal generator of the one parameter Lie group of transformations

$$
\begin{aligned}
& x^{*}=X(x, u, \varepsilon), \\
& u^{*}=U(x, u ; \varepsilon) .
\end{aligned}
$$

Let

$$
\begin{gathered}
X^{(k)}=\xi_{i}(x, u) \frac{\partial}{\partial x_{i}}+\eta(x, u) \frac{\partial}{\partial u}+\eta_{i}{ }^{(l)}\left(x, u, u_{1}\right) \frac{\partial}{\partial u_{1}}+\cdots+ \\
\eta^{(k)}{ }_{i_{1}, i_{2}, \ldots, i_{k}}\left(x, u, u_{1}, u_{2}, \cdots, u_{k}\right) \frac{\partial}{\partial u_{i_{1}, i_{2}, \ldots, i_{k}}}
\end{gathered}
$$

be the corresponding $k$ th extended infinitesimal generator of (3) where $\eta_{i}^{\prime}=\eta_{i}^{\prime}(x, u)$ is given by

$$
\eta_{i}^{\prime}=D_{i} \eta-\left(D_{i} \xi_{i}\right) u_{j}, \quad i=1,2, \cdots, n ;
$$

and $\quad \eta_{i_{1}, i_{2}, \cdots, i_{j}}^{j}$ is given by

$$
\eta_{i_{1}, i_{2}, \cdots, i_{k}}^{j}=D_{i_{k}} \eta_{i_{1}}^{(k-1)},_{i_{2}}, \ldots, i_{k-1}-\left(D_{i_{k}} \xi_{j}\right) u_{i_{1}, i_{2}, \ldots i_{k-1} j}
$$

$i_{j}=1,2, \cdots, n$ for $j=1,2, \ldots, k$ with $k=1,2, \ldots$ interms of $(\xi(x, u), \eta(x, u))$. Then one parameter Lie group of transformations (4-6) is admitted by the partial differential equation (2) iff

$$
\left.X^{(k)} F\left(x, u_{1} u_{1}, u_{2}, \cdots, u_{k}\right)\right|_{F=0}=0
$$




\section{Classical Lie Symmetries of the GBE4 Equation}

We consider the one parameter Lie point transformations of $(t, x, u)$ given by

$$
\begin{aligned}
& t^{*}=t+\varepsilon \xi_{1}(t, x, u)+O\left(\varepsilon^{2}\right) \\
& x^{*}=x+\varepsilon \xi_{2}(t, x, u)+O\left(\varepsilon^{2}\right) \\
& u^{*}=u+\varepsilon \eta_{1}(t, x, u)+O\left(\varepsilon^{2}\right) .
\end{aligned}
$$

The GBE4 equation

$$
u_{t}=a u_{x:}+b u_{x x x x}+c\left(u^{2}\right)_{x x}
$$

is invariant under this transformation if it is invariant under the operator

$$
X=\xi_{1} \frac{\partial}{\partial t}+\xi_{2} \frac{\partial}{\partial x}+\eta_{1} \frac{\partial}{\partial u}+\varsigma_{11} \frac{\partial}{u_{t t}}+\varsigma_{22} \frac{\partial}{\partial u_{x x}}+\gamma_{2222} \frac{\partial}{\partial u_{x x x x}}
$$

Hence $\xi_{1}, \xi_{2}$ and $\eta_{1}$ are found from the determining equations derived from the invariancy condition (9) [7].

To determine the classical Lie symmetries of the GBE4 equation, we first start the computer algebra system MATHEMATICA. Then we call the package Lie [] by

$1=:<<$ Lie.m

and use the gbe 4 .dgl file prepaped

Indep $\operatorname{Var}=\{\mathrm{t}, \mathrm{x}\}$

DependVar $=\{u\}$

Eqlist $=-\left\{D[u[t, x],\{t, 2\}]+a * D[u[t, x],\{x, 2\}]+b * D[u[t, x],\{x, 4\}]+c * D[u[t, x], x]^{\wedge} 2+\right.$ $\left.\mathrm{c}^{*} \mathrm{u}[\mathrm{t}, \mathrm{x}] * \mathrm{D}[\mathrm{u}[\mathrm{t}, \mathrm{x}],\{\mathrm{x}, 2\}]\right\}$;

Subslist $=\mathrm{D}[\mathrm{u}[\mathrm{t}, \mathrm{x}],\{\mathrm{x}, 4\}]$;

ParameterS $=\{\}$;

List $X i=\{x i 1[t, x, u], x i 2[t, x, u]\}$;

ListPhi $=\{$ fil $[t, x, u]\}$;

by running

\section{2=:Lie["gbe4.dgl",Info-> True]}

The determining equation as given in the output, consits of 31 linear partial differential equations.

Rewritten in the usual style, the infinitesimal generators of the finite subgroup of the symmetry group of the GBE4 equation are given by

$$
X_{1}=\frac{\partial}{\partial t}, \quad X_{2}=\frac{\partial}{\partial x}, \quad X_{3}=2 t \frac{\partial}{\partial t}+x \frac{\partial}{\partial x}-\left(\frac{a}{c}+2 u\right) \frac{\partial}{\partial u} .
$$

The nonvanishing commutators are 


$$
\left[X_{1}, X_{2}\right]=2 X_{1}, \quad\left[X_{2} X_{3}\right]=-X_{2} .
$$

Hence a commutator table can be constructed as follows:

$$
\begin{array}{cccc} 
& X_{1} & X_{2} & X_{3} \\
X_{1} & 0 & 0 & 2 X_{1} \\
X_{2} & 0 & 0 & -X_{2} \\
X_{3} & -2 X_{1} & X_{2} & 0
\end{array}
$$

The linear space $\left\{X_{1}, X_{2}, X_{3}\right\}$ spanned by $X_{1}, X_{2}, X_{3}$ is a Lie algebra with the skew symmetric operator in the table. The subspaces $L_{2}=\left\{X_{1}, X_{2}\right\}$ and $L_{1}=\left\{X_{1}\right\}$ are two and one dimensional subspaces of $L_{3}=\left\{X_{1}, X_{2}, X_{3}\right\}$ respectively. Furthermore these subalgebras have the inclusion property

$$
L_{1} \subset L_{2} \subset L_{3}
$$

and hence $L_{3}$ is a solvable Lie algebra.

\section{Similarity Reductions of the GBE4 Equation}

Similarity reduction corresponding the symmetry generator $X_{2}+\frac{1}{\gamma} X_{1}$ then obtained by solving the characteristic equations. The similarity variables are

$$
u=w(\varsigma), \quad \varsigma=x-y t
$$

and $w(\varsigma)$ satisfies the fourth order ordinary differential equation:

$$
b w^{(i v)}+\left(a-\gamma^{2}\right) w^{\prime \prime}+c\left(w^{2}\right)^{n}=0, \quad{ }^{\prime}=\frac{d}{d \varsigma}
$$

where $\gamma$ the speed of the traveling wave is an arbitrary constant. The ODE in (17) has the only symmetry due to the translation invariance along the $\varsigma$ - axis and this equation can be integrated to get the second order ODE

$$
\mathbf{b} w^{\prime \prime}+\left(a-\gamma^{2}\right) w+c w^{2}=C_{1} \varsigma+C_{2}
$$

For $C_{l}=0$, multiplying both sides by $w^{\prime}$ the equation (18) can be reduced to the quadrature

$$
\int \frac{d w}{\sqrt{-\frac{2 c}{3 b} w^{3}+\frac{\gamma^{2}-a}{b} w^{2}+\frac{2 C_{2}}{b} w+C_{3}}}=\varsigma+C_{4} \text {. }
$$

For $C_{3}=0$ and $\gamma^{2}=a$ one obtains the solitary wave solution

$$
W=\frac{3 C_{2}}{c} \operatorname{Sech}^{2}\left(\sqrt{\frac{C_{2}}{2 b}}\left(x-\sqrt{a} t+C_{4}\right)\right) .
$$


The similarity generator $X_{3}$ yields the similarity variables

$$
u=t^{-1} w(\varsigma)-\frac{2 a}{c}, \quad \varsigma=x t^{-1 / 2}
$$

and $w(\varsigma)$ satisfies the fourth order ordinary differential equation:

$$
b w^{(i v)}-c\left(w^{2}\right)^{\prime \prime}-2(\varsigma w)^{\prime}+\varsigma\left(w^{\prime}-\varsigma w^{\prime \prime}\right) / 4=0, \quad '=\frac{d}{d \varsigma}
$$

This equation has no Lie point symmetries at all.

\section{REFERENCES}

[1] LIE, S., Vorlesungen Über Differentialgleichungen mit Bekannten Infinitesimalen Transformationen, Teube, Leipzig, (1891) (Printed again by Chelsea, New York (1967).

[2] WINTERNITZ, P., What is new in the study of differential equations by group theoretical methods?, In Symmetries and Nonlinear Phenomena, D. Levi and P. Winternitz (Eds.), 229-248, World Scientific, Singapore, (1988).

[3] BAUMANN, G., Lie symmetries of differential equations. A Mathematica program to determine Lie symmetries, Preprint (1996).

[4] SCHWARZ, F., Symmetries of differential equations. From Sophus Lie to computer

algebra, Siam Review, V. 30 (3), 450-481, (1988).

[5] VOROB'EV, E. M., Symmetry analysis of nonlinear PDE's with Mathematica program "SYMMAN", Nonlinear Math. Phys. 3 (1-2), 68-84, (1996).

[6] OLVER, P. J., Applications of Lie Groups to Differential Equations, Springer, London (1994).

[7] IBRAGIMOV, N. H., Lie Group Analysis of Differential Equations, CRC Press, London (1994) 